\title{
Continuous wave four-wave mixing at 2 micron in Chalcogenide microstructured fiber
}

\author{
Davide Grassani *, Sida Xing, Svyatoslav Kharitonov, Adrien Billat, Steevy Cordette, Armand Vedadi, Camille \\ Sophie Brès \\ Ecole Polytechnique Fédérale de Lausanne (EPFL), Photonic Systems Laboratory (PHOSL), STI-IEL, Station 11, CH-1015 Lausanne, \\ Switzerland \\ *davide.grassani@epfl.ch
}

\begin{abstract}
We present the first demonstration of continuous-wave four-wave mixing at $2 \mu \mathrm{m}$ in short chalcogenide microstructured fiber. Idlers over approximately $15 \mathrm{~nm}$ on anti-Stokes side of the pump were measured for a coupled pump power of $94 \mathrm{~mW}$.

OCIS codes: (060.5295) Photonic crystal fibers; (190.4380) Nonlinear optics, four-wave mixing
\end{abstract}

\section{Introduction}

With the growing interest in the 2 micron band and the mid-infrared (MIR), there is an increasing development of sources, platforms and waveguides operating in such spectral regions. Platforms capable of supporting nonlinear interactions are interesting and important as the use of nonlinear effects is a powerful technique to achieve all-optical ultrafast processing and to generate light at targeted wavelengths in a tunable way through four-wave mixing (FWM). For such applications FWM with record gain and bandwidth has been achieved in silica highly nonlinear fibers with pumping in the telecommunication band [1]. However the silica suffers from excessive losses beyond $2 \mu \mathrm{m}$ and novel materials have to be considered for processing in the short wave infrared (SWIR) and MIR. On the one hand silicon is considered to be a great candidate: silicon waveguide have small effective area, high Kerr index leading to a strong nonlinearity. Also, while in the telecom band silicon suffers from two photon absorption, such effect is significantly reduced at $2 \mu \mathrm{m}$. Several demonstrations of FWM and comb generation have been recently reported using such approach. On the other hand, chalcogenide microstructured fibers are also been of great interest [2] owing to their transparency in the infrared region and intrinsic nonlinearity 1000 time higher than silica. In addition, microstructured fibers can offer a lot of freedom in terms of dispersion engineering, an essential ingredient for efficient FWM, through control of its geometry. Finally, as chalcogenide can be draw in fiber and owing to the recent progress in fabrication, long interaction length can be relied upon.

Demonstration of continuous wave (CW) FWM in the telecom band [3] as well as generation of MIR supercontinuum using high peak power pumping in a chalcogenide microstructured fiber [4] has been report. Here, we report for the first time, to the best of our knowledge, the demonstration of FWM at 2 micron in such fiber. A continuous wave pump together with a tunable continuous wave signal gives rise to idler waves over about $15 \mathrm{~nm}$ with only $94 \mathrm{~mW}$ of pump power. Peak conversion efficiency (CE) of $-36 \mathrm{~dB}$ is measured.

\section{Experiment Setup}

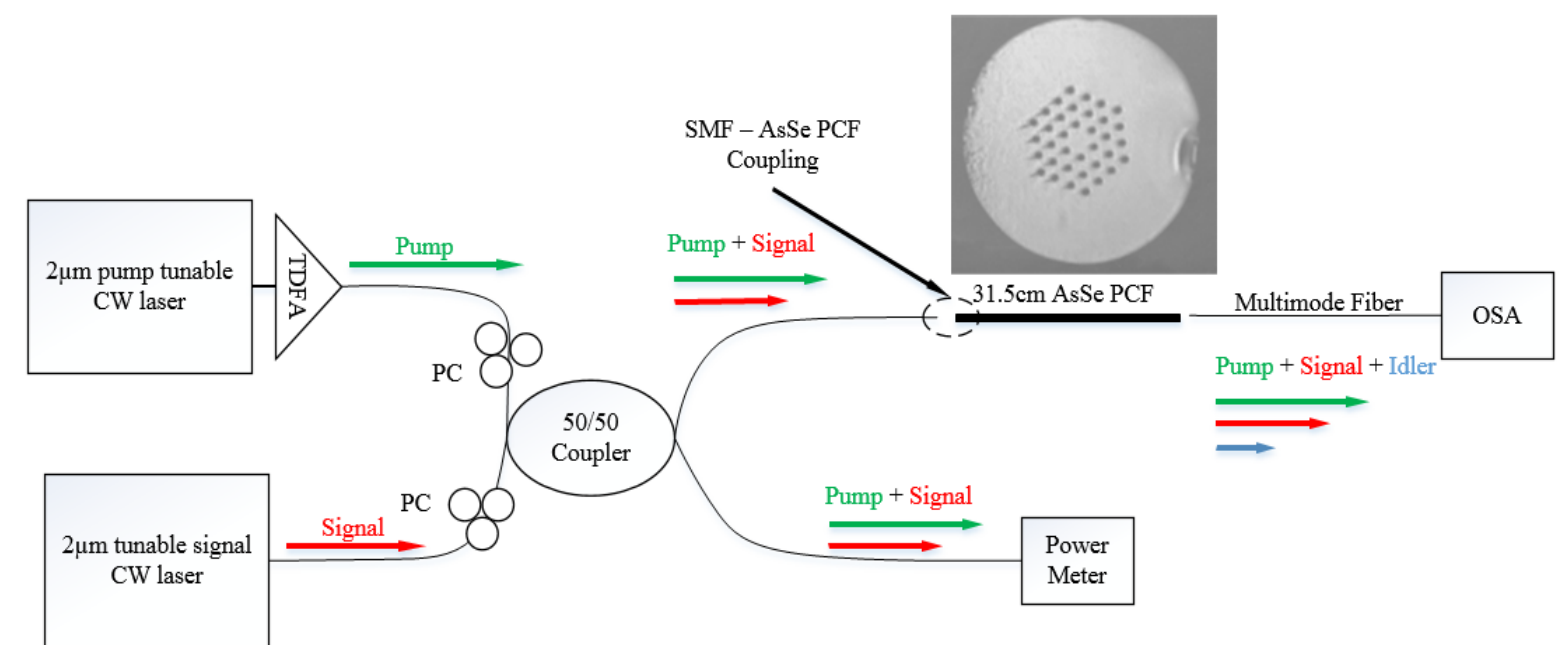

Fig. 1. Experimental setup. TDFA: Erbium doped fiber amplifier; PC: polarization controller; PCF: photonic crystal fiber; OSA: optical spectrum analyzer; PM: power meter.

The experimental setup is shown in figure 2. Two polarized tunable Thulium (Tm) doped CW fiber lasers built by our group [5] were used as the pump and signal. The pump laser relied on a ring cavity design embedding a $4 \mathrm{~m}$ long Thulium fiber pumped at $1600 \mathrm{~nm}$ and a fixed fiber Bragg grating at $1980 \mathrm{~nm}$ and $0.5 \mathrm{~nm}$ wide. The signal laser is also a ring cavity with $11.5 \mathrm{~m}$ Thulium double sided pumped at $1600 \mathrm{~nm}$ and a tunable grating filter (2 nm wide). A Thulium-doped fiber amplifier (TDFA) was used to boost the power of the pump laser. Due to lack of an additional suitable filter, the amplified spontaneous emission (ASE) could not be removed, slightly limiting the sensitivity of the 
idler measurements. After passing both signal and pump lasers through polarization controllers (PC), they were combined by a 50/50 coupler. At one arm of the coupler output, a power meter was connected to monitor the powers. The other output of coupler went directly into the chalcogenide microstructured sample. The $31.5 \mathrm{~cm}-\mathrm{long}_{\mathrm{S}} \mathrm{As}_{38} \mathrm{Se}_{62}$ microstructured fiber was cleaved and a cleaved SMF-28 fiber was used for butt coupling the pump and signal. The chalcogenide fiber facet was inspected by a camera as shown in figure 1 . The alignment was performed with a low power $1.55 \mu \mathrm{m}$ laser and input coupling losses were measured to be approximately $5 \mathrm{~dB}$. Minor adjustments were then performed to optimize the coupling at $2 \mu \mathrm{m}$. The output of the fiber was then once again butt coupled to a multimode fiber connected to the optical spectrum analyzer (OSA),

The AsSe fiber under test (FUT) was provided by PERFOS. A scanning electron microscope (SEM) was used to get a precise measurement of its geometry structure. The air hole diameter $d$ and the pitch distance $\Lambda$ were measured to be about $3.79 \mu \mathrm{m}$ and $8.54 \mu \mathrm{m}$, respectively. This gives a ratio of 0.44 between air hole diameter and pitch distance.

\section{Results}

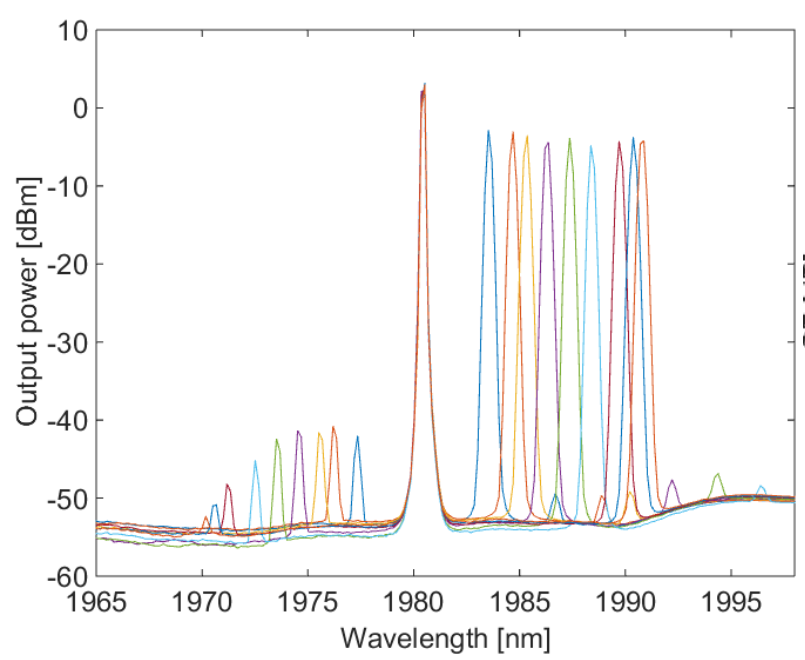

Fig. 2 FWM Spectra for different signal wavelengths

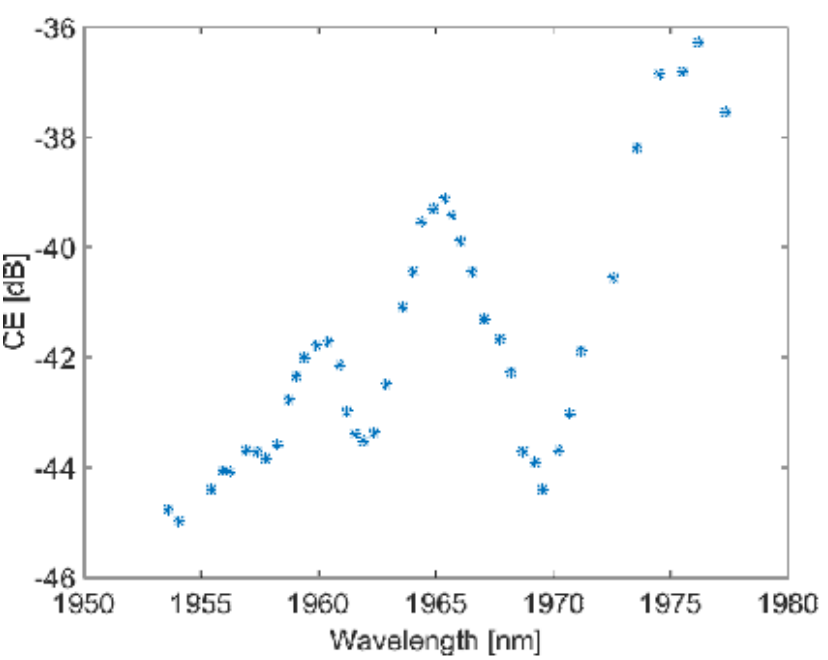

Fig. 3 Conversion Efficiency as a function of the generated idler wavelength for $94 \mathrm{~mW}$ of coupled pump power.

In order to assess the nonlinear optical properties of the fiber we performed two kinds of FWM measurements on the FUT. In a first experiment we kept the power of both the pump and signal lasers constant. The pump wavelength was fixed at $1980 \mathrm{~nm}$ while the signal wavelength was swept over a range of about $25 \mathrm{~nm}$. The pump and signal power of 94 $\mathrm{mW}$ and $41 \mathrm{~mW}$ respectively was chosen as the best compromise between idler signal to noise ratio (SNR) and safe operating conditions to avoid fiber melting. Fig. 2 shows the superimposed optical spectra for some signal wavelengths measured near the pump. Idlers are generated on the anti-Stokes sides of the pump. Additionally, some wave generation is also observed on the Stokes side of the signal. Note that we deliberately a higher base line level and limited the detection of the idlers when the signal was tuned to low wavelength. This is only a limitation of our setup and can be optimized with a more adequate filtering of the pump. Due to the large core diameter, our chalcogenide PCF is highly dispersive in the $2 \mathrm{um}$ region and multiple period of oscillations of the idler generation were observed. Therefore, considering the low pumping level used, we can safely neglect the nonlinear contribution to the phase mismatch. Within this approximation the phase mismatch is thus $\Delta \mathrm{k}=2 \pi \lambda^{2} \Delta \omega^{2} \mathrm{D} / \mathrm{c}$, where $\mathrm{D}$ is the dispersion parameter and $\Delta \omega$ the detuning from the pump frequency. The data in Fig.3, showing the conversion efficiency $\left(\mathrm{CE}=\mathrm{P}_{\text {idl }} / \mathrm{P}_{\text {sig }}\right.$ with $\mathrm{P}_{\text {idl }}$ the measured idler power and $\mathrm{P}_{\text {sig }}$ the signal power) as a function of the idler wavelength, were thus employed to retrieve the group velocity dispersion of the FUT near the pump wavelength. The obtained value is $\mathrm{D} \approx-336(\mathrm{ps} / \mathrm{nm} / \mathrm{km})$.

We estimated the nonlinear parameter $\gamma$ from a second experiment, in which we fixed the signal detuning at about 6 $\mathrm{nm}$ from the pump wavelength and we recover the FWM spectra for different pump powers. As expected, the CE as a function of the pump power follows a quadratic behavior, as it is shown in Fig 4. Considering a propagation loss coefficient $\alpha \approx 0.3 \mathrm{~dB} / \mathrm{m}$ estimated from linear transmission measurements, and the group velocity dispersion value obtained in the first experiment, we extracted a nonlinear coefficient $\gamma \approx 470\left(\mathrm{~W}^{-1} \mathrm{Km}^{-1}\right)$. Both the dispersion parameter and the nonlinear coefficient are in good agreement with the theoretical values expected from the geometry and composition of the fiber. It shows that the strong normal dispersion limits the four-wave mixing efficiency. 


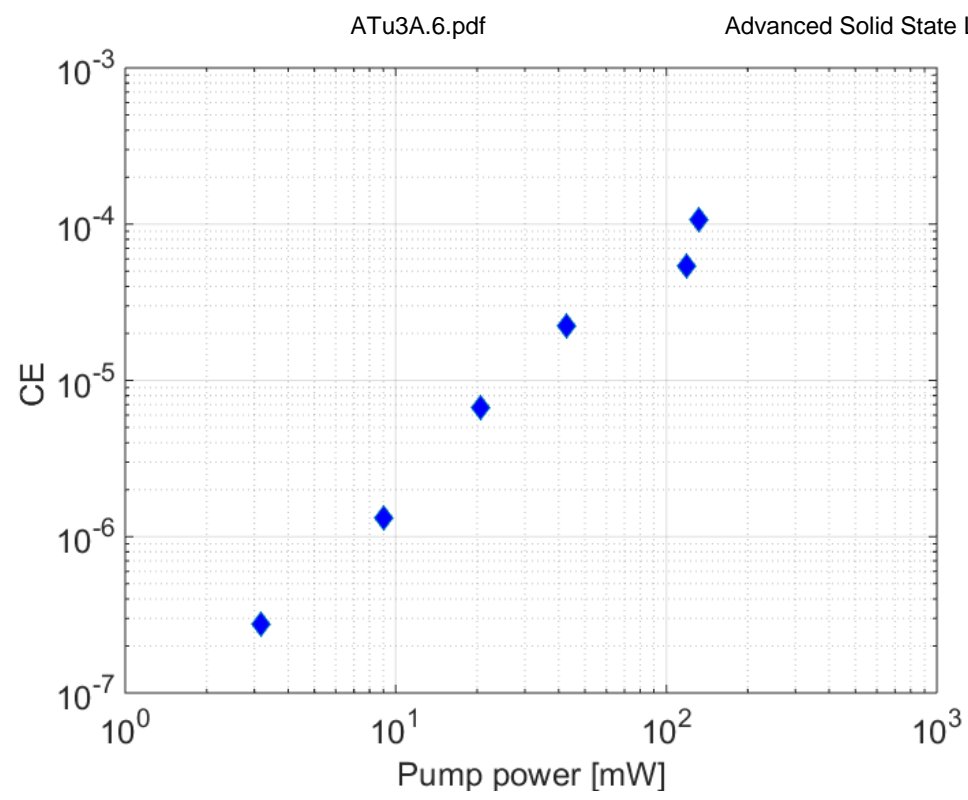

Fig. $4 \mathrm{CE}$ as a function of the pump power at a detuning of $6 \mathrm{~nm}$ from the pump wavelength.

In conclusion we demonstrated for the first time $\mathrm{CW}$ four-wave mixing in the $2 \mu \mathrm{m}$ wavelength region in a chalcogenide microstructured fiber by using two self-made Thulium based tunable fiber lasers. Only off-the shelf telecommunications components were required. The dispersion parameter and the nonlinear coefficient are in good agreement with the results found in literature taking into account for the different fiber geometry and the wavelength range, showing that chalcogenide microstructured fiber can be successfully employed as a platform for the 2 micron band. A maximum conversion efficiency of $-36 \mathrm{~dB}$ was measured with only $94 \mathrm{~mW}$ of pump power owing to the high nonlinearities and despite the large normal dispersion. These results are therefore very encouraging: by optimizing the fiber geometry in order to blue shift the zero dispersion wavelength around 2 micron with a suspended core or tapered design [6] we can expect to significantly increase the conversion efficiency and the bandwidth of the four wave mixing process. With such optimized design, one can envision an all fiber chalcogenide based wavelength tunable laser for the mid infrared.

We acknowledge PERFOS for fabricating the fiber. This work is supported in part by the European Research Council under grant agreement ERC-2012-StG 306630-MATISSE

\section{References}

[1 T. Torounidis and P. Andrekson, "Broadband single-pumped fiber-optic parametric amplifiers," Photonics Technology Letters, IEEE 19, 650-652 (2007).

[2] J. M. Dudley and J. R. Taylor, "Ten years of nonlinear optics in photonic crystal fibre," Nat Photon 3, 85-90 (2009).

[3] C. Brès, S. Zlatanovic, A. O. J. Wiberg, and S. Radic, "Continuous-wave four-wave mixing in cm-long Chalcogenide microstructured fiber," Optics Express 19, B621-B627 (2011).

[4] O. Mouawad, J. Picot-Clémente, F. Amrani, C. Strutynski, J. Fatome, B. Kibler, F. Désévédavy, G. Gadret, J.-C. Jules, D. Deng, Y. Ohishi, and F. Smektala, "3.5- $\mu \mathrm{m}$ bandwidth mid-infrared supercontinuum generation in a 2-cm long suspended-core chalcogenide fiber," in Advanced Photonics, OSA Technical Digest (online) (Optical Society of America, 2014), SoTu2B.2.

[5] S. Kharitonov, A. Billat, L. Zulliger, S. Cordette, A. Vedadi, and C.-S. Brès, "Kerr nonlinearity of Thulium-doped fiber near $2 \mu \mathrm{m}, "$ in $C L E O$ : Science and Innovations, (Optical Society of America, 2015), JTu5A. 31.

[6] Marcin Szpulak, S. Février, 'Chalcogenide $\mathrm{As}_{2} \mathrm{~S}_{3}$ Suspended Core Fiber for Mid-IR Wavelength Conversion Based on Degenerate Four-Wave Mixing,' IEEE Photonics Technol. Lett. 21 (13), pp 884 (2009). 